\title{
NORMAL FORMS FOR SKEW-SYMMETRIC MATRICES AND HAMILTONIAN SYSTEMS WITH FIRST INTEGRALS LINEAR IN MOMENTA
}

\author{
G. THOMPSON
}

(Communicated by Kenneth R. Meyer)

\begin{abstract}
Using an appropriate notion of equivalence, those classical Hamiltonian systems which admit a first integral of motion polynomial of degree one in momentum are classified. The classification is effected by means of finding a normal form for a skew-symmetric matrix under the action of orthogonal symmetry.
\end{abstract}

1. Introduction. The past few years has seen something of a revival in the study of completely integrable Hamiltonian systems. In this paper I am concerned with one aspect of integrability. Specifically, I ask whether it is possible to characterize classical Hamiltonian systems which have at least one first integral which is a homogeneous polynomial of degree one in momenta. Several results along these lines were obtained even in the nineteenth century (see the references given by Whittaker [7]). Indeed in 1901, Darboux [2] considered the problem of characterizing all classical systems with two degrees of freedom which have a first integral which is quadratic in momenta. Darboux's results were reproduced almost verbatim by Whittaker [7] and more recently, several authors gave a complete solution to the problem $[\mathbf{3}, \mathbf{5}, \mathbf{6}]$. Thereafter followed many papers which gave examples of systems with two degrees of freedom having a first integral polynomial in momenta. (See the recent survey by Hietarinta [4].)

In spite of these successes, very little systematic work has been undertaken for systems with three or more degrees of freedom. This is probably because the conditions one obtains when looking for a first integral are extremely complicated. Nevertheless, I shall show below that it is possible to give a complete characterization of those Hamiltonian systems which admit the simplest and most geometrically significant of all first integrals, namely, those which are degree one polynomials in momenta. The main technique used to obtain this characterization depends on finding a normal form for a skew-symmetric matrix under the action of orthogonal similarity. This normal form is presented in $\S 2$.

In $\S 3$ I formulate with some care the meaning of equivalence for classical Hamiltonian systems. Since we are attempting a classification problem, it is essential to do this. The classification proper is carried out in $\S 4$.

2. Normal forms for skew-symmetric matrices. Let $\Omega$ and $\Lambda$ be $2 n \times 2 n$ real skew-symmetric matrices. Then of course $\Omega$ and $\Lambda$ are said to be congruent, if

Received by the editors February 5, 1987 and, in revised form, May 18, 1987.

1980 Mathematics Subject Classification (1985 Revision). Primary 58F07; Secondary 15 A21. 
there is $A \in G L(2 n, \mathbf{R})$ such that

$$
A^{t} \Lambda A=\Omega \text {. }
$$

The normal form for $\Omega$ under congruence is very well known [1] and I shall rederive it by an alternative method shortly. It is even more well known that a real symmetric matrix can be diagonalized by an orthogonal matrix with its eigenvalues appearing on the diagonal. It would seem natural therefore to ask what is the normal form of a skew-symmetric matrix under orthogonal similarity (of course the notions of similarity and congruence concur for the orthogonal group). Let us note that a skew-symmetric matrix $\Omega$ has either zero or purely imaginary eigenvalues and that if $\mu$ is an eigenvalue, so is $\bar{\mu}$.

PROPOSITION 2.1. Given the $2 n \times 2 n$ skew-symmetric real matrix $\Omega$, there exists $A \in O(2 n)$ such that $A^{t} \Omega A$ is given by

$$
A^{t} \Omega A=\left[\begin{array}{cccccccccccc}
0 & \lambda_{1} & 0 & 0 & 0 & \ldots & 0 & 0 & 0 & 0 & \ldots & 0 \\
-\lambda_{1} & 0 & 0 & 0 & 0 & & 0 & 0 & & & & \\
0 & 0 & 0 & \lambda_{2} & & & & 0 & & & & \\
0 & 0 & -\lambda_{2} & 0 & & & & & & & & \\
& & & & & & & 0 & & & & \\
& & & & & & 0 & \lambda_{r} & & & & \\
0 & \ldots & & & & & -\lambda_{r} & 0 & 0 & \ldots & 0 \\
0 & \ldots & & & & & & 0 & 0 & \ldots & 0 \\
0 & \ldots & & & & & & 0 & 0 & \ldots & 0
\end{array}\right]
$$

where the eigenvalues of $\Omega$ are $\pm \lambda_{1} i, \ldots, \pm \lambda_{r} i, 0$ (with multiplicity $2(n-r)$ ) and $2_{r}$ is the rank of $\Omega$.

PROOF. Identify $\mathbf{R}^{2 n}$ with $\mathbf{C}^{n}$ in the usual way (compare [1, Theorem 3.1.19 ff]). Then there exists a (possibly degenerate inner product $G$ on $\mathbf{R}^{2 n}$ and a (possibly degenerate) Hermitian inner product $H$ on $\mathbf{C}^{n}$ such that

$$
H=G+i \Omega \text {. }
$$

Now since $H$ is Hermitian, there is an element $A$ of $U(n)$ such that $A^{+} H A$ is diagonal, with the eigenvalues of $H$ appearing on the diagonal. Moreover, $\lambda$ is an eigenvalue of $H$ iff $\pm \lambda i$ is an eigenvalue of $\Omega$. If we now reidentify $\mathbf{C}^{n}$ with $\mathbf{R}^{2 n}$ and identify $U(n)$ as a subgroup of $O(2 n)$, we find that $A^{t} \Omega A$ is precisely of the form given by (2.2).

COROllaRY 2.2. There exists $A \in G L(n, \mathbf{R})$ such that $A^{t} \Omega A$ is of the form given by (2.2) in which, in addition, each of the $\lambda_{i}$ have the value unity.

PROOF. If $\lambda$ is nonzero, the $2 \times 2$ matrix $\left(\begin{array}{cc}0 & \lambda \\ -\lambda & 0\end{array}\right)$ is congruent to $\left(\begin{array}{cc}0 & 1 \\ -1 & 0\end{array}\right)$. Indeed, if $B \in G L(2, \mathbf{R})$ and has determinant $1 / \lambda$,

$$
B^{t}\left(\begin{array}{cc}
0 & \lambda \\
-\lambda & 0
\end{array}\right) B=\left(\begin{array}{cc}
0 & 1 \\
-1 & 0
\end{array}\right) \text {. }
$$

To reduce $\Omega$ to the form stated, simply build up a matrix with $r$ such $B$ 's, 1's and 0 's in the obvious way.

The point of Corollary 2.2 is that it shows that we do not have to go through the usual lengthy argument to find the normal form of a skew-symmetric matrix acted 
on by congruence. It can essentially be deduced from the diagonalization procedure for Hermitian matrices (compare [1, Proposition 3.1.2 (ii)]).

I show now how to extend Proposition 2.1 to cover the case of skew-symmetric matrices with an odd number of rows and columns.

PROPOSITION 2.3. Given the $\left(2 n_{1}\right) \times(2 n+1)$ skew-symmetric real matrix $\Omega$ of rank $2 n$, there exists $A \in O(2 n+1)$ such that $A^{t} \Omega A$ is given by

$$
A^{t} \Omega A=\left[\begin{array}{cccccccccc}
0 & \lambda_{1} & 0 & 0 & 0 & \ldots & 0 & 0 & 0 & \\
-\lambda_{1} & 0 & 0 & 0 & & & & & \vdots \\
0 & 0 & 0 & \lambda_{2} & & & & & & \\
\cdot & & -\lambda_{2} & 0 & & & & & & \\
\cdot & & & & & & & & \\
\cdot & & & & & & & & \\
& & & & & & & & \\
& & & & & & & 0 & \lambda_{n} & \\
0 & \ldots & & & & & 0 & 0 & 0 & 0 \\
0 & & & & & & 0 & 0 & 0 & 0
\end{array}\right]
$$

ProOF. Write $\Omega$ as $\left[\begin{array}{cc}M & q \\ -q^{t} & 0\end{array}\right]$ where $M$ is an invertible $2 n \times 2 n$ skew-symmetric matrix and $q$ is a column $2 n$-vector. Consider the $(2 n+1) \times(2 n+1)$ matrix $\left[\begin{array}{ll}A & b \\ c & a\end{array}\right]$ where $A$ is a $2 n \times 2 n$ matrix, $b$ and $c$ are $2 n$ column and row $2 n$-vectors, respectively, and $a$ is a scalar. In fact, choose $c$ to be $q^{t} M^{-1} /\left(1+\left\|M^{-1} q\right\|^{2}\right)^{1 / 2}$ and $a$ to be $1 /\left(1+\left\|M^{-1} q\right\|^{2}\right)^{1 / 2}$, where $\left\|M^{-1} q\right\|$ is the Euclidean length of the column vector $M^{-1} q$. Further, choose $A^{-1}$ be be the positive 'square root' of the $2 n \times 2 n$ positive operator $I_{2 n}+M^{-1} q\left(M^{-1} q\right)^{t}$ and $b$ to be $A M^{-1} q$. Then one may verify that $\left(\begin{array}{ll}A & b \\ c & a\end{array}\right)$ defines an element of $O(2 n+1)$ and that

$$
\left[\begin{array}{ll}
A & b \\
c & a
\end{array}\right]\left[\begin{array}{cc}
M & q \\
-q^{t} & 0
\end{array}\right]\left[\begin{array}{cc}
A & b \\
c & a
\end{array}\right]^{t}=\left[\begin{array}{cc}
A \widetilde{M} A^{t} & 0 \\
0 & 0
\end{array}\right]
$$

where $\widetilde{M}=M-M^{-1} q q^{t}+\left(M^{-1} q q^{t}\right)^{t}$. The result now follows by applying Proposition 2.1 to $\widetilde{M}$.

LEMMA 2.4. The $(2 n+1) \times(2 n+1)$ skew-symmetric matrix

$$
\left[\begin{array}{ll}
O_{2 n} & q \\
-q^{t} & 0
\end{array}\right]
$$

where $q$ is a $2 n$ column vector, is orthogonally similar to

$$
\left[\begin{array}{lc}
O_{2 n} & 0 \\
& \\
& \vdots \\
& 0 \\
0 \ldots 0-\lambda & 0
\end{array}\right] .
$$

ProOF. Note that if $A \in O(2 n),\left(\begin{array}{ll}A & 0 \\ 0 & 1\end{array}\right) \in O(2 n+1)$ and that

$$
\left[\begin{array}{cc}
A & 0 \\
0 & 1
\end{array}\right] \Omega\left[\begin{array}{cc}
A & 0 \\
0 & 1
\end{array}\right]^{t}=\left[\begin{array}{cc}
O_{2 n} & A q \\
-q^{t} A^{t} & 0
\end{array}\right] .
$$

Thus, we can choose $A$ so that $(A q)^{t}=(0,0, \ldots, 0, \lambda)$ for some $\lambda \in \mathbf{R}$.

The preceding results can be combined to yield the following theorem. 
THEOREM 2.5. A skew-symmetric $k \times k$ matrix $\Omega$ is orthogonally similar to a matrix of the form given by (2.2). Furthermore, there are precisely $r$ nonzero $\lambda_{i}$ 's where $2 r$ is the rank of $\Omega$ and the eigenvalues of $\Omega$ are $\pm \lambda_{1} i, \ldots, \pm \lambda_{r} i$ and 0 (with multiplicity $k-2 r$ ).

PROOF. The case of $k$ even is Proposition 2.1 so we may assume $k$ is odd, say, $k=2 n+1$. In fact, write

$$
\Omega=\left[\begin{array}{cc}
M & q \\
-q^{t} & 0
\end{array}\right],
$$

much in the same way as in Proposition 2.3 and assume $q \neq 0$ (otherwise the theorem is already proved). Now by applying Proposition 2.1 to $M$ and using some permutation matrices which define elements of $O(2 n+1)$, we may assume that $\Omega$ is orthogonally similar to a matrix of the form

$$
\left[\begin{array}{cccc}
O_{2(n-r+1)} & & 0 & p \\
0 & \Lambda & & s \\
-p^{t} & & -s^{t} & 0
\end{array}\right]
$$

where $\Lambda$ is a skew-symmetric matrix of rank $2(r-1)$ and $p$ and $s$ are column vectors of lengths $2(n-r+1)$ and $2(r-1)$, respectively.

Now by applying Proposition (2.3) to (2.6) we may assume that $s=0$ and by further permutations that $\Omega$ is orthogonally similar to a matrix of the form

$$
\left[\begin{array}{ccc}
\Lambda & 0 & 0 \\
0 & O_{2(n-r+1)} & p \\
0 & -p^{t} & 0
\end{array}\right] .
$$

The proof of the theorem is finally completed by applying Lemma 2.4 to the $(2(n-r)+3) \times(2(n-r)+3)$ matrix $\left[\begin{array}{cc}O_{2(n-r+1)} & p \\ -p^{t} & 0\end{array}\right]$ and using more permutations.

3. The meaning of equivalence for Hamiltonian systems. Let $M$ be a smooth $m$-manifold $T^{*} M$ its cotangent bundle and $\pi: T^{*} M \rightarrow M$ the canonical submersion. I consider Hamiltonian functions $h: T^{*} M \rightarrow \mathbf{R}$ of the form

$$
h=\frac{1}{2} G(p, p)+\left(\pi^{*} V\right)(p)
$$

where $G$ is a contravariant Riemannian metric on $M$ (dual to a covariant metric $g$ ), $V: M \rightarrow \mathbf{R}$ is the potential energy function and $p$ a typical point of $T^{*} M$. Now a system of local coordinates $\left(x^{i}\right)$ on $T^{*} M$ gives rise to an adapted coordinate system $\left(x^{i}, p_{i}\right)$ on $T^{*} M$. If the metric $g$ is assumed to be flat, then $h$ can be written locally and more familiarly in the form

$$
h=\frac{1}{2} \delta^{i j} p_{i} p_{j}+V\left(x^{i}\right)
$$

where $\left(x^{i}, p_{i}\right)$ is an adapted coordinate system on $T^{*} M$. (Here and throughout the summation convention applies on repeated indices.) However, it is important to emphasize that (3.2) is an accurate representation of (3.1) if and only if $\left(x^{i}\right)$ is a coordinate system relative to which $G$ is represented by $\delta^{i j}$.

Suppose that we are given two Hamiltonians $h_{1}$ and $h_{2}$ on $T^{*} M$ of the form (3.1), whose associated Riemannian metrics are flat. Suppose further that with respect to each of these metrics $M$ is complete as a metric space and is also simply connected. Then each of the metrics make $M$ into an affine inner product space. 
(More precisely, $M$ is diffeomorphic to the affine space $\mathbf{R}^{m}$ and each tangent space $T_{x} M$ is an inner product space which is isometric to the inner product space on the corresponding tangent space in $\mathbf{R}^{m}$ with the standard Euclidean metric. A choice of origin in $M$, which we shall modify if necessary, thus makes $M$ itself into an inner product space; see [8].) The Hamiltonians $h_{1}=\frac{1}{2} G_{1}\left(p_{1} p\right)+\left(\pi^{*} V_{1}\right)(p)$, $h_{2}=\frac{1}{2} G_{2}\left(p_{1} p\right)+\left(\pi^{*} V_{2}\right)(p)$ will then be said to be equivalent, if there exists a diffeomorphism $\phi$ of $M$ such that $\phi^{*} G_{2}=G_{1}$ and $\phi^{*} V_{2}=V_{1}$. Thus $\phi$ defines an isometry of the inner product structures on $M$ and naturally $\tilde{\phi}^{*} h_{2}=h_{1}$, where $\tilde{\phi}$ is the diffeomorphism of $T^{*} M$ induced by $\phi$. Furthermore, if $\left(x^{i}\right)$ and $\left(\bar{x}^{j}\right)$ are coordinates on $M$ relative to which the components of $G_{1}$ and $G_{2}$ are $\delta_{i j}$, the transformation $\phi$ will be of the form

$$
\bar{x}^{i}=A_{j}^{i} x^{j}+b^{i}
$$

where $A \in O(m)$ and $b \in \mathbf{R}^{m}$.

One could of course envisage weaker notions of equivalence of Hamiltonians than that just introduced; for example, if $\Phi$ is a symplectic diffeomorphism (canonical transformation) such that $\Phi^{*} h_{2}=h_{1}$; or again, if besides the latter condition, $\Phi$ is the lift $\tilde{\Phi}$ to $T^{*} M$ of some diffeomorphism $\phi$ of $M$. However, these weaker notions of equivalence lead to results of a mainly theoretical nature because they do not preserve the familiar local appearance of a Hamiltonian as given by (3.2).

4. Mechanical Hamiltonians with linear integrals of motion. In this section I turn to the main concern of the paper, namely, Hamiltonians with first integrals which are linear in momenta. For the remainder of the paper I shall be concerned only with Hamiltonians of the form (3.1) for which $g$ is flat.

Given $h$ of the form (3.1), it is well known and easy to show that a first integral of the dynamical system determined by $h$ which is a homogeneous polynomial of degree one in momenta corresponds to a Killing vector field of the metric $g$ on $M$ ( $g$ being the covariant metric dual to $G$ of 3.1). In addition, $K$ satisfies

$$
K V=0
$$

or, in local coordinates,

$$
K^{i} \frac{\partial V}{\partial x^{i}}=0
$$

(This assertion follows from Noether's theorem [1] and indeed the first integral is precisely $K$ itself when viewed as a real-valued function linear in $p$ on $T^{*} M$.)

Now in $\mathbf{R}^{m}$ and using standard coordinates $\left(x^{i}\right)$, any Killing vector $K$ consists simply of a constant linear combination of rotation $R_{i j}=x^{i}\left(\partial / \partial x^{j}\right)-x^{j}\left(\partial / \partial x^{i}\right)$ and translation fields $T_{i}=\partial / \partial x^{i}$. In fact write

$$
K=a_{i j} R_{i j}+\alpha_{i} T_{i}
$$

where $\alpha_{i} \in R$ and $a_{i j}$ is an $m \times m$ matrix which, without loss of generality, may be assumed to be skew-symmetric.

Consider next the effect of applying the isometric transformation (3.3) to $K$. Indeed if $K$ is transformed into $\bar{K}$ and we write

$$
\bar{K}=\bar{a}_{i j} \bar{R}_{i j}+\bar{\alpha}_{i} \bar{T}_{i}
$$


then one finds that $a_{i j}$ and $\overline{a_{i j}}$ and $\alpha_{i}$ and $\bar{\alpha}_{i}$, respectively, are related by

$$
\begin{gathered}
a_{k l}=\bar{a}_{i j} A_{i k} A_{j l}, \\
\alpha_{k}=2 \bar{a}_{i j} b_{i} A_{j k}+\bar{\alpha}_{i} A_{i k} .
\end{gathered}
$$

(4.5) says that the matrix $\bar{a}_{i j}$ is acted on by orthogonal similarity and (4.6) that some of the $\alpha_{k}$ 's can be normalized to zero by choosing the $b_{i}$ 's appropriately. Specifically, suppose that $\bar{a}_{i j}$ has rank $2 r$ and invoke Theorem (2.5); then $a_{i j}$ may be assumed to be of the form given by (2.2). Thereafter, choosing $A_{j k}$ to be $\delta_{j k}$ in (4.6) we have

$$
\alpha_{k}=2 a_{i j} b_{i}+\bar{\alpha}_{k}
$$

Since $a_{i j}$ is assumed to be given by $(2.2)$, we can choose $b_{1}, \ldots, b_{2 r}$ such that $\alpha_{1}, \cdots, \alpha_{2 r}$ are zero. In short, we can normalize $K$ into the form

$$
\begin{aligned}
K= & \lambda_{1}\left(x^{1} \frac{\partial}{\partial x^{2}}-x^{2} \frac{\partial}{\partial x^{1}}\right)+\lambda_{2}\left(x^{3} \frac{\partial}{\partial x^{4}}-x^{4} \frac{\partial}{\partial x^{3}}\right) \\
& +\cdots+\lambda_{r}\left(x^{2 r-1} \frac{\partial}{\partial x^{2 r}}-x^{2 r} \frac{\partial}{\partial x^{2 r-1}}\right)+b_{2 r+1} \frac{\partial}{\partial x^{2 r+1}}+\cdots+b_{m} \frac{\partial}{\partial x^{m}}
\end{aligned}
$$

Let us now reconsider (4.6) in the light of the normalization which has already been carried out. In fact we may write

$$
A=\left[\begin{array}{cc}
I_{2 r} & 0 \\
0 & B
\end{array}\right] \quad \text { where } B \in O(n-2 r)
$$

and since $a_{i j}=0$ for $i, j \geq 2 r+1,(4.6)$ enables us to further normalize $K$ as

$$
\begin{aligned}
K= & \lambda_{1}\left(x^{1} \frac{\partial}{\partial x^{2}}-x^{2} \frac{\partial}{\partial x^{1}}\right)+\lambda_{2}\left(x^{3} \frac{\partial}{\partial x^{4}}-x^{4} \frac{\partial}{\partial x^{3}}\right) \\
& +\cdots+\lambda_{r}\left(x^{2 r-1} \frac{\partial}{\partial x^{2 r}}-x^{2 r} \frac{\partial}{\partial x^{2 r-1}}\right)+\mu \frac{\partial}{\partial x^{2 r+1}}
\end{aligned}
$$

for some $\mu \in \mathbf{R}$.

The partial differential equation (4.1) (or (4.2)) with $K$ given by (4.9) can be integrated in closed form by the method of characteristics, yielding the solution as an arbitrary smooth function of $m-1$ arguments. I summarize the results in the form of the following theorem:

THEOREM 4.1. Let $h: T^{*} M \rightarrow \mathbf{R}$ be a Hamiltonian of the form (3.1) with $G$ a flat metric. Then the Hamiltonian flow determined by $h$ has a first integral $I$ which is polynomial of degree one in momenta, iff $h$ can be represented locally as $h=\frac{1}{2} \delta^{i j} p_{i} p_{j}+V$, where $V$ is an arbitrary smooth function of the following $m-1$ arguments ( $r$ being some integer satisfying $0 \leq r \leq[m / 2],[m / 2]$ denoting 
the integral part of $m / 2)$ :

$$
\left(x^{1}\right)^{2}+\left(x^{2}\right)^{2},\left(x^{3}\right)^{2}+\left(x^{4}\right)^{2}, \ldots,\left(x^{2 r-1}\right)^{2}+\left(x^{2 r}\right)^{2}
$$

$\lambda_{2} \arctan \left(\frac{x^{2}}{x^{1}}\right)-\lambda_{1} \arctan \left(\frac{x^{4}}{x^{3}}\right), \lambda_{3} \arctan \left(\frac{x^{4}}{x^{3}}\right)-\lambda_{2} \arctan \left(\frac{x^{6}}{x^{5}}\right), \ldots$,

$$
\begin{gathered}
\lambda_{r} \arctan \left(\frac{x^{2 r-2}}{x^{2 r-3}}\right)-\lambda_{r-1} \arctan \left(\frac{x^{2 r}}{x^{2 r-1}}\right) ; \\
\left(\lambda_{1}+\lambda_{2}+\cdots+\lambda_{r}\right) x^{2 r+1}-\mu\left(\arctan \frac{x^{2}}{x^{1}}+\arctan \frac{x^{4}}{x^{3}}+\cdots+\arctan 3 \frac{x^{2 r}}{x^{2 r-1}}\right) ; \\
x^{2 r+2}, x^{2 r+3}, \ldots, x^{m-1}, x^{m} .
\end{gathered}
$$

Furthermore, in that case $I$ is given by

$$
I=\lambda_{1}\left(x^{1} p_{2}-x^{2} p_{1}\right)+\cdots+\lambda_{r}\left(x^{2 r-1} p_{2 r}-x^{2 r} p_{2 r-1}\right)+\mu p_{2 r+1} .
$$

ACKnowledgments. The author is grateful to Tim Swift for helpful discussions during the course of this work and to the Science and Engineering Research Council for support in the form of a Research Fellowship.

\section{REFERENCES}

1. R. Abraham and J. E. Marsden, Foundations of mechanics, Benjamin, Reading, Mass., 1978.

2. G. Darboux, Archives Neerlandaises 6 (1901), 371.

3. B. Dorizzi, B. Grammaticos and A. Ramani, A new class of integrable systems, J. Math. Phys. 24 (1983), 2282-2288.

4. J. Hietarinta, Direct methods for the search of the second invariant, preprint, Univ. of Turku, 1986.

5. A. Ankiewicz and C. Pask, The complete Whittaker theorem for two-dimensional integrable systems and its application, J. Phys. A 16 (1983), 4203-4208.

6. G. Thompson, Darboux's problem of quadratic integrals, J. Phys. A 17 (1984), 985-988.

7. E. T. Whittaker, $A$ treatise on the analytical dynamics of particles and rigid bodies, 4th ed., Cambridge Univ. Press, Cambridge, 1959.

8. J. A. Wolf, Spaces of constant curvature, Publish or Perish, Boston, Mass., 1974.

Department of MAThematics, UNIVERSity Of EDINBURGh, EDINBURGH, EH9 3JZ, UNITED KINGDOM 